Data Hand ling Division $\mathrm{DD} / 87 / 8$

February 1987

Two years of real progress in European HEP networking:

A CERN perspective

B.E. Carpenter, F. Flückiger, J.M. Gerard, D. Lord, B. Segal 



\title{
TWO YEARS OF REAL PROGRESS IN EUROPEAN HEP NETWORKING: A CERN PERSPECTIVE
}

\author{
B.E.Carpenter, F.Fluckiger, J.M.Gerard, D.Lord, B.Segal \\ Data Handling Division \\ CERN \\ 1211 Geneva 23 \\ Switzerland
}

\section{INTRODUCTION}

A lot has happened in networking in the last two years. It is premature to speak of maturity, but there are signs of the end of infancy. We can note two main symptoms of this change: the increasing importance of industrially supported solutions, and the increasing dependency of working physicists on networks. For members of the highly international and widely dispersed LEP collaborations, these two trends have had a dramatic effect on the working environment. Indeed, it is difficult to conceive how the collaborations could function otherwise.

In this paper, we report on recent evolution in networking facilities for European high energy physicists. Although the main emphasis is on wide-area networks (WANs), we start with a review of developments in local-area networks (LANs) using the example of the CERN site. We then review the growth in WAN infrastructure in the last two years before turning to progress in the high-level services for physicists, and to PTT issues. The conclusion attempts to draw some lessons and to predict some future trends.

\section{LOCAL AREA NETWORKS}

CERN is interesting as a case study for several reasons. There are particular regions on the CERN site which contain large concentrations of computers, so the necd for LANs was seen very early. The computers are of a wide variety of types and operating systems, thus requiring that the LANs not be restricted to one particular make of computer. The whole site is large enough that one single LAN is not feasible, so that high speed LAN interconnection is necessary. Finally, the international character of CERN requires computers on LANs to be able to access other computers all over the world.

\subsection{Local Area Network hardware and Installation}

There have been many different LANs installed in CERN in the past decade. Some were developed at CERN for special purposes, although CERNET is completely general purpose. None could claim to be a standard (in the ISO sense).

The first real standard was ISO $8802 / 3$, commonly known as Ethernet. The first Ethernet installation dates from 1983, a pilot Ethernet installed in the computer centre. It has grown to such an extent that it is now as complex, and with as many types of user, as one is likely to find. Extension of this Ethemet throughout the adjacent office building has taken place over the past two years. It is now complete, and includes fan-out units, multiport repeaters, thin Ethernet segments and a spine of standard Ethemet cable. It now has about 100 computers connected. The fact that the number is not known exactly is inherent to Ethernet philosophy; new users can simply connect onto an existing transceiver, fan-out unit or thin Ethernet without assistance. 
The last two years has seen a rapid growth in the number of Ethernets at CERN, so that there are now about a dozen. Most of these are classified as public access, and it has been found best to have a central team of hardware experts.

In addition to Ethernet there are now two other standard LANs emerging, ISO 8802/4 (Token Bus) and $8802 / 5$ (Token Ring). The Token Ring is of interest, because it is strongly supported by IBM and because it has been chosen for the LEP control system. Experience is limited so far, since the product is so much more recent than Ethernet. Nonetheless, the fact that the computer centre capacity is currently largely IBM or IBM-compatible, plus the 300 IBM Personal Computers already at CERN, suggest that Token Rings might be installed in a wider context than accelerator controls. One of the questions for the near future is whether a single LAN can suffice to serve a particular region, or whether one must cable some areas for several LANs.

\subsection{LAN interconnection}

The products made by industry for interconnecting I ANs at high speed are point-to-point connections (mainly between Ethernets). These may be simple local or remote repeaters, or intelligent bridges which only forward packets when necessary. Where the distance between the Ethernets exceeds three kilometres, the transmission speed can today only be a fraction of the raw Ethernet speed. Because of the distances at CERN, the number of I,ANs to be interconnected, and traffic estimates, it was already clear three years ago that no solution based only upon point-to-point connections could satisfy our requirements. An analogy is that individual computers were interconnected by point-to-point links in the early days, but later by networks: now the LANs themselves are getting so numerous that they themselves need networking.

The availability of CERNET, covering the site with links of at least $2 \mathrm{Mbits} / \mathrm{s}$, led to a solution based on units called Frigates, which are 68000-based VME crates, each connecting an Ethemet to CERNET. Every Ethernet has one Frigate, which forwards Ethernet packets to remote Ethernets, via CERNET, on the basis of the physical destination address. This service is equivalent to that offered by commercial Ethernet bridges, but better adapted to CERN geography. This solution has some limitation in speed; we await new industrial technology in order to improve performance. For all practical purposes, CERN's twelve Ethernets behave as one large Ethernet.

At present we anticipate that non-Ethernet LANs, including Token Ring, will be interconnected to Ethernet by protocol gateways rather than by bridges, with a corresponding lack of generality.

\subsection{Protocols and interworking}

Although international standard (OSI) protocols are not yet mature, the arrival of LANs has brought in de facto standards. At CERN two are important, TCP/IP and DECnet, and they have overtaken plans to use CERNET protocols on Ethernet.

DECnet protocols have been used at CERN for a long time, but their use across Ethernet is recent. There are now over 50 VAXes on the Ethernets, permitting simple and efficient multi-computer working. In fact, DECnet traffic makes up much of the total Ethernet traffic.

Where interworking of different computer makes is required, TCP/IP protocols have caught on. Their great advantage is that they exist for computers ranging from the $\mathrm{PC}$ to the mainframe, and that they generally work with a minimum of problems, although with less functionality than DECnet. Their use is normally but not exclusively for file transfer or remote login. They have been chosen for the LEP control system. 
Another approach to the interworking problem is the use of industrially supported units implementing a protocol or gatewaying two protocols. Examples of the first at CERN include WISCNET (TCP/IP on the IBM) and Interlink (DECnet on the IBM). Examples of the second at CERN include the DECnet SNA gateway. These solutions are highly effective, although somewhat expensive. In some cases, they allow a user to communicate with unfamiliar computers using familiar techniques (e.g. a VAX user communicating with the IBM via DECnet!).

An emerging trend is a preference for avoiding the traditional circuit-switch approach for terminals. Now that PC clones are inexpensive, there is a move towards the use of networked PCs as terminals, with a suitable protocol. For dumb terminals we now benefit from the presence of LANs by installing terminal concentrators, especially in new buildings equipped from the start with Ethernet. A recently identified requirement is for interworking between the circuit-switch equipment (too expensive to throw away) and the new LAN world. Although CERN is installing a new digital telephone system with data switching capability, we are unsure whether this will be a major factor in data traffic.

\subsection{Where next?}

Over the past two years we have learncd how to install, interconnect, and operate $L \Lambda N$ in a flexible manner. The end users have got used to having networking available. We have realised the need for products which allow the connection of LANs to each other, to Wide Area Networks and to the terminal switching systems. In some cases we already have such products installed.

A new type of networking, which one may call "fourth generation" networking, is appearing on LANs. This is truly distributed computing, using techniques such as remote procedure call (RPC) and remote file access (as opposed to simple file transfer). Such techniques have been used in accelerator control systems for a number of years, but are now entering wider service. An existing example is the new CERN electronic mail directory, actually an Oracle data base on a VAX interrogated by RPC from IBM VM/CMS, VAX VMS, Unix, IBM-PC, and Apollo systems.

An encouraging recent development is the acquisition of an OSI Transport Protocol Class 4 (TP4) implementation in a portable form, 1 which will be used in conjunction with the VOTS product for the VAX to provide program-to-program communication between microprocessors and VAXes, via Ethernet, in several experiments. We are also evaluating a TP4-based implementation of NETBIOS for personal computers on Ethernet. Considerable thought has gone into a CERN-standard calling sequence for TP4. Coupled with the OSI migration plans announced for DECnet and anticipated for the TCP/IP world, these developments give serious hope of eventual convergence among LAN protocols and of convergence with WAN protocols.

\section{WIDE AREA INFRASTRUCTURE}

\subsection{Infrastructure for communication with CERN}

This section concentrates on the infrastructure set up for the wide area communications between CERN and the other physics institutes and laboratories.

1 The OSIAM_C product marketed by MARBEN INFORMATIQUE in Lurope and by OMNICOM in the USA. 


\subsubsection{Evolution of the infrastructure since 1985}

The CERN infrastructure for wide area communication is based on the use of three types of communication medium: general leased lines, EARN leased lines, and Public Packet Switching Networks (PPSN) using the X.25 protocol. The last two years have been characterized by the increased number and bandwidth of leased lines, and by the achievement of full European connectivity with public X.25.

\subsubsection{General Leased Lines}

CERN is connected today to six European or American institutes by leased lines at speeds ranging from 9.6 to $19.2 \mathrm{kbps}$ : CEN-Saclay (F), IN2P3-Lyons (F), RAL-Oxford (UK), LAPP-Annecy (F), INFN-Bologna (I) (2 lines) and MIT-Boston (USA, for the LEP L3 experiment). The Lyons and Boston lines as well as the second line to Bologna have been set up during the last 18 months. The bandwidth of 4 of these 7 lines has also been increased by the use of new modems, and the upgrading of the 3 other lines is foreseen for 1987. The result is that, since 1985, the aggregate bandwidth available between CERN and other institutes via leased lines has been more than doubled.

Preparatory work has also started for the connection of 4 additional leased lines in 1987: University of Geneva, SIN-Zurich, ETH-Zurich and FNAL. All these new lines will run at 56 or $64 \mathrm{kbps}$, and most of the existing lines are also likely to be upgraded to this speed in the course of 1987.

It is important to note the general tendency to exploit these lines with the X.25 protocol. Currently, 8 of the 11 lines mentioned are or will be using X.25, which offers the external institutes the advantages of a wide range of standard or proprietary higher level services.

\subsubsection{EARN infrastructure}

The CERN node of EARN is now a major international node: the number of lines has been multipied by a factor 3 over the last two years, and has reached a total of 12 ( 7 international and 5 Swiss lines). There are five EARN end-nodes at CERN. The EARN-BITNET-NETNORTIl network is now estimated to give access to about 550 sites and 1800 hosts. The traffic through the CERN international node is one order of magnitude greater than two years ago. In the short term the infrastructure will be improved by increasing the speed of the main international lines to $14.9 \mathrm{kbps}$, and using a $56 \mathrm{kbps}$ link between EARN and BITNET. In the longer term, the evolution of the EARN infrastructure will depend on the solutions chosen for the migration to ISO standards.

\subsubsection{X.25 Infrastructure}

The CERN X.25 infrastucture to connect to the Swiss PPSN has not been substantially modified, but more reliable equipment has been incorporated. In addition, the on-site X.25 switching capacity has been increased by one order of magnitude over the last two years.

\subsubsection{Problems and future trends for the WAN infrastructure}

The major problem which has affected and will continue for the next two years to affect the CERN WAN infrastructure is the inability to establish a satisfactory solution for the LAN-WAN interconnection. As said before, the tendency is to use X.25 more and more over leased lines, and this protocol is effectively mandatory to access sites not reachable via leased lines. On site, Ethernet is now the favoured carrier. The fact that some high level packages (Coloured Books) normally run over X.25, and the lack of general purpose equipment for Ethernet-X.25 interconnection, obliges CERN to operate an interim on-site X.25 network to give off-site access to CERN hosts. This network, which has now reached about 30 host connections, satisfies connectivity requirements but entails extra costs and manpower, as well as a multiplication of the communication interfaces required on the CERN hosts. 
This problem should find a final solution when the universal use of the ISO Transport Protocol is achieved. At this stage, transport level relays between Ethernet and X.25 will be commercially available, unfortunately probably needing a protocol conversion between TP4 used over the LAN and TP2 used, at least in Europe, over the WAN. This is not expected to happen before two years. In the meantime partial solutions will be installed for special services (remote logon) or proprietary protocols (DECnet), which should stabilize and later reduce the size of the CERN local X.25 network. Once satisfactorily achieved, the LAN-WAN interconnection should allow the X.25 infrastructure of all institutes to be limited to the connection to the PPSN and the leased lines.

Another difficulty is that the X.25 PPSNs have already started to show their limits. Designed mainly for interactive access and transactional applications, their tarifs and bandwidth, especially between countries, are unsuitable for more demanding applications (file transfers, remote graphics, etc.). However, no alternative yet exists yet with comparable connectivity. This is why CERN foresees continued increase in the X.25 leased lines to the larger or closer institutes. Smaller institutes will continue to communicate via the X.25 PPSNs, and CERN will probably have to double its connection to the Swiss TELEPAC network before the end of the decade. X.25 PPSNs will also continue to carry an ever increasing electronic mail traffic, for which their bandwidth limitations are less visible.

For the 1990s, the ISDN is attractive, as it is expected to offer the only long-term switched services (except some limited switched satellite services) at speeds of the order of $64 \mathrm{kbps}$. (Note that the European PTTs have no consistent plan to upgrade the X.25 PPSN to such speeds, although it would be technically possible). CERN will study ISDN issues during 1987; a number of questions are still open. The first set of questions concerns the success of ISDN in itself: will the PTTs be able to respect their announced timeframe (which refers to 1988 for first availability), and will the manufacturers integrate ISDN facilities in their products? The second set of uncertainties concerns how ISDN services will relate to existing networks: transparent level 1 connections between X.25 installations, internal X.25 services provided between ISDN installations, etc.? The third set of questions concerns the role that such a medium has to play for an organization like CERN: we already said that most of the big laboratories will probably prefer to connect to CERN via leased lines; ISDN could be the solution for medium size institutes, but the inherent characteristics of circuit switching, i.e. the inability to use a given channel with more than one partner site, will restrict the number of concurrently connected institutes to 2 with the basic interface and 30 with the primary ratc interface. Finally, the tariff structures are unknown and may prove to be prohibitive.

\subsection{Infrastructure for the rest of HEP communication}

For their national communications, European HEP physicists often use the facilities offered by the general purpose national academic and research (A\&R) networks. They also make extensive use of EARN. Only the Italian (with INFNET) and recently the French (with PHYNET) physicists have set up their own dedicated networks. Both use a leased line infrastructure (running DLCnet in Italy and X.25 in France). Apart from lincs to CERN, there are to our knowledge no international HEP leased lines in Europe except one from RAL to DESY, and one planned from Italy to the USA.

Other HEP physicists, as mentioned, use or plan to use their national general purpose A\&R network. The major event during the last two years has been the emergence of national projects, and in some cases implementations, in almost all the European countries, thus following the example given by JANET in the UK and later DFN in Germany: UNA in Austria, Centernet in Denmark, FUNET in Finland, REUNIR in France, ARIADNE in Greece, HEANET in Ireland, SURFNET in the Netherlands, UNINETT in Norway, IRIS in Spain, SUNET in Sweden, SWITCH in Switzerland. As far as infrastructure is concerned, almost all these new networks have decided to use public X.25. This is even true for those offering DECnet as the first available service (SURFNET, SUNET) despite certain inconveniences when running DECnet over an X.25 PPSN. JANET, designed 10 years ago, remains the only major national A\&R network based on a mesh of leased lines, but it also uses the X.25 protocols. 
The tendency for non-CERN HEP communication is therefore to use public switched services, primarily the PTT X.25 networks. This is mainly derived from the policy currently followed by national (and international) general purpose A\&R networks, and despite the recognized limitations of the public $\mathrm{X} .25$ networks and their inadequacy for applications more demanding than remote login or electronic mail. But this is somehow in contradiction with the tendency also observed in favour of leased lines to connect big or nearby institutes to CERN, and with the enormous success of EARN. This contradiction reflects the current lack of adequate offerings from the European PTTs especially in the range of medium and high speed, and the resulting difficulty for user communities like HEP to define a fully consistent strategy for long distance communication infrastructure.

\section{WIDE AREA NETWORK SERVICES}

\subsection{Evolution of the Services since 1985}

Five phenomena, not all with the same weight, can be noted:

- the explosion of DECnet

- the continuous growth of EARN

- the creation of the RARE association and the COSINE project

- the introduction of OSI services (for X.400 electronic mail)

- the installation of application level gateways at CERN

Remote login using X.29 over X.25 is not discussed, but has continued to be a universal basic service in Europe. A limited growth in the use of the JANET "Coloured Book" protocols outside the UK is also not discussed in detail.

\subsubsection{DECnet}

The generalization of the use of DEC computers for HEP became dominant in 1985, especially for the LEP collaborations. Since Europe had no infrastrusture for long distance TCP/IP services, only two networking architectures could offer the services needed by the collaborations: the "Coloured Book" protocols and DECnet. One major collaboration, L3, decided after evaluation to use a mixture of these architectures. Others decided to implement only DECnet (and to use EARN as appropriate).

The implementation of the first DECnet connections revealed the existence of a considerable potential connectivity, since several HEP (INFNET, US HEPNET) or research (SPAN) organizations had already set up DECnet networks. This connectivity was also greatly improved by the establishment of leased lines between CERN and these networks (INFN-Bologna, L3-MIT). In the meantime, the connectvity offered by the Coloured Books (JANET, part of L3, PHYNET in France, several sites in Furope), although important, seemed less promising. It therefore turned out that DECnet has been installed over the last two years on virtually all the HEP DEC machines, sometimes in conjunction with the Coloured Books (and sometimes TCP/IP for IAN use, or JNET for EARN).

In addition to the potential connectivity, two other reasons for the success of DECnet are its functionality, judged as very satisfactory by the physicists, and its ability to run over a wide range of media (DDCMP, Ethernet, X.25), thus simplifying the LAN-WAN interconnection problem.

DECnet links, either over leased lines or X.25 PPSNs exist between CERN and a large number of European Countries (Austria, Finland, France, Italy, Portugal, Spain, Switzerland, and soon the Netherlands, Sweden, ...), whilst the US HEPNET and SP $A N$ are accessible via the L 3 link between CERN and MIT. In total, more than 1000 nodes are probably interconnected through this amorphous world wide network. A first meeting of the managers of the European HEP DECnet will be organized this month at CERN to establish a minimal framework for operation and management, including coordination with the American physics DECnets. 
The official policy of all the national A\&R networks, and of the "European HEP networking community" (ECFA Subgroup 5) is to migrate as soon as possible to ISO OSI standards. DECnet should be one of the first manufacturer architectures to migrate to OSI, and this is viewed as a great advantage.

\subsubsection{EARN}

EARN continued to grow steadily over the last two years, and is now available in 21 European countries. It still offers the two main services of interpersonal file transfer and electronic mail which have not been recently substantially modified. However, during 1985-86 EARN introduced new services on the network. The most noticeable is the NETSERV facility, a set of information centres available in almost all the countries. Also, the LISTSERV facility offers enhanced capabiities for mail distribution lists. At CERN, a gateway has been developed between EARN and the MVS WYLBUR environment. EARN intends to provide computer conferencing, for which a number of alternatives are under study.

The overall connectivity of $\mathrm{E} \Lambda \mathrm{RN}$ has also been improved by the use of a number of RSCS emulation packages. With 35 such emulators now available, openness of EARN to non-IBM machines has been achieved (740 VAXes are part of EARN-BITNET).

\subsubsection{RARE and COSINE}

RARE (Réseaux Associés pour la Recherche Européenc) is an association formally created in June 1986 which federates European national academic and research networks. RARE also admits, with a special status, international organizations like CERN, ECFA, and EARN. The objective of RARE is the creation and the promotion of Europe wide data communication services for the academic and research community. COSINE is a project, part of the European EUREKA programme, with essentially the same objective as RARE. COSINE receives funding from the European governments which are part of EUREKA. RARE will prepare, through its working groups and a central secretariat, the specifications of COSINE. RARE and COSINE are strongly supported by the European Economic Community (EEC), which sees them as a market push for the European Information Technology industry. RARE and COSINE have decided to use the PTT public switching infrastructure, starting with the public X.25 networks, and not to make use of leased lines. The policy is to provide services based on ISO OSI standards.

RARE and COSINE are of interest for HEP because they have created a technical and political climate in Europe for the launching of national $\Lambda \& R$ networks. These should become directly usable by the physicists for their domestic communications. Except for the pioneers (UK, Germany, the Nordic countries) all the national network projects have been triggered or accelerated by the development of RARE and COSINE. Futhermore, the international COSINE services will be themselves, when available, directly usable by the physicists. It is noteworthy that HEP is by far the strongest, the most demanding, and the best organized of the communities likely to use the RARE/COSINE services.

\subsubsection{First OSI services for Electronic Mail}

Migrating to the use of OSI standards is the policy of RARE and COSINE (not to mention EARN), and it is also the policy of CERN and of the European HEP community (ECFA Subgroup 5). In the process of this major migration, the most important event has been the introduction of a pre-OSI service for electronic mail in Europe during 1985-86. This service is based on the use of the EAN package from the University of British Columbia, which is an almost complete implementation of the X.400 standard.

Initiated by CERN, University College (London), DFN and UNINETT, this service grew rapidly, despite a lack of funding for its central administration. This service is now receiving the support of RARE and the EEC which are to finance its management. The next version of $E \Lambda N$ is expected to bc fully compatible with X.400. 
The EAN service now covers 13 European countries (Denmark, France, Finland, Iceland, Ireland, Italy, the Netherlands, Norway, Portugal, Spain, Sweden, Switzerland, and the UK). There are links to Australia, Canada, and the USA. About 80 nodes are connected in Europe (including 10 at CERN) after one year of existence, and 150 nodes world-wide. A total connectivity is achieved by the existence of number of gateways giving access to many mail networks: ARPA (COM, EDU, GOV, MIL, ORG), BITNET, CSNET, EARN, EUNET, JANET, MAILNET, MLNET, OZ and USENET.

Another precursor of migration to OSI standards for mail is the release of the DEC X.400 product. This is being evaluated in several places in Europe, including CERN. It is also relevant to mention the experiment intiated in 1986 between several EARN sites with an X.400 package developed by the IBM Networking Centre in IIcidelberg. This experiment, using the public X.25 networks, will continue in 1987.

\subsubsection{Provision of Application level Gateways at CERN}

Like many other organizations, CERN identified in the period 1984-85 an increasing need for protocol translation in order to allow interworking between interim protocols and smooth transition to the OSI protocols. This gave rise to a development programme for application level converters which led to the release of the GIFT service in 1985 and the MINT service in 1986.

GIFT is a joint project aiming at developing and operating a multiple file transfer protocol converter. The collaboration initially included CERN, INFN, RAL and Oxford University. The first version of the system operating at CERN allows on-the-fly protocol conversion between any pair of the following file transfer protocols: DECnet, CERNET and JANET (Blue Book). The collaboration has recently been extended to include SARA (Amsterdam) which will contribute a TCP/IP FTP slot to the whole system, and DFN plans an HMI-RDA slot. Preliminary work has also started to incorporate a OSI FTAM slot, with an industrial collaborator. GIFT is heavily used for file transfers from Italy (using DECnet) and the UK (using JANET) to the CERN MVS system, but also for exchange of files through CERN between Italy and the UK.

MINT is a CERN project for the development of a set of protocol converters between mail protocols used on the CERN site. The MINT system is composed of a central multiple converter complemented by several satellite converters. The central converter, implemented on a UNIX machine, allows cross conversion between the EARN, UUCP and EAN mail protocols. The satellite converters include a gateway between DECnet mail and EAN, and a gateway between EARN and WYLBUR. Complemented by outside gateways (EAN to JANET in the UK, and BIINET to the other US mail networks in the US $\Lambda$ ), this infrastructure offers a full mail connectivity to CERN users. This concentration of gateways giving access to virtually all the existing mail networks seems to be unique in Europe, and CER $N$ has been requested by many institutes to open its conversion services to part or all of the European A\&R community. Although flattering, this request poses obvious operational and financial problems.

\subsection{Problems and Future Trends}

The main problem that the HEP community and CERN are facing for long distance communication services is the appearance of a jungle of protocols: EARN-RSCS, DECnet, JANET-Coloured Books, SNA, etc. This imposes on all the HEP centres the need for skilled staff to install and support some or all of these protocols, as well as considerable effort to develop and operate converters between them. But provided these resources can be made available, the final IHEP user is provided with a relatively satisfactory overall service.

A second range of problems affect these interim networks themselves. The management of DECnet has become difficult, due to the size reached by the international network, addressing limitations (the 
area code problem), and the fact that no resources are easily available to establish some form of proper centralized administration. Attempts are therefore being made, on a best effort basis, to solve the most crucial management problems. EARN is provided with a relatively satisfactory central management, but the technical future of the network is not yet clear. At the launching of EARN the PTTs insisted on a committment to move to ISO standards and this is implicit in EARN's membership of RARE. However, it is technically unclear whether this would concern the lower, higher, or all the levels. Although promising, the experimental X.400 implementation on VM/CMS is a pilot exercise, and seems far from a service likely to replace RSCS.

If one looks at the short and medium term future, the protocol jungle for wide area communication has probably reached its worst. TCP/IP protocols are not expected to be used over WANs in the European HEP' community. (This is not technically excluded, and could change if several supercomputers were to be installed in Europe by HEP before OSI migration is complete.)

DECnet with its current phase IV protocols will continue to grow over the coming two years, but the Coloured Books have probably reached their maximum of extension. The next essential milestone in the transition process towards the OSI world will be the release of DECnet phase V which is expected to be OSI compatible. (It is inconvenient that this release has a high chance of coinciding with the commissioning of the LEP experiments.) This will mark the real beginning of the generalization of migration to OSI. In the meantime, experience will have been gained by the use OSI application packages (X.400, and FTAM later) to mount pilot experiments or pre-services. Protocol converters will still be needed between interim protocols over the next 3 years. Later, only converters between interim and OSI protocols should be required. These converters will by then probably be used with the OSI protocols serving as intermediate relays between older protocols, for a period of a minimum two years. This scenario assumes that no unsolvable problems will appear in the areas where ISO standardization is incomplete, such as distributed directories, naming registration, and network management.

\section{PTT ISSUES}

Except in Britain, the European common carriers are monopolies with restrictive rules and unhelpful tariff structures. In particular, third-party traffic switching is generally disallowed, and there are moves toward volume-dependent charging on leased lines. Fortunately, CERN has obtained partial relaxation by the Swiss PTT of the third party switching prohibition. CERN is now authorized to switch third party traffic between leased lines through its high level protocol converters (GIFT and MINT), but of course only in relation to research activitics.

A related problem for EARN is its financing when IBM stops its partial funding in January 1988. It is indeed recognized by the European HEP (and wider A\&R) community that a functionality at least equal to that of EARN must be maintained; its loss would be a catastrophe for the LEP experiments. Funds have therefore to be sought to cover line rental, and the maintenance of the nodes funded by IBM. A particularly delicate and confusing parameter of the overall bill may be the cost of the lines, since some PTTs have expressed an intention to apply volume dependent charges in addition to the rental. However, recently a number of PTTs have showed signs of a more liberal attitude to regulatory problems and have indicated that such charging will not be imposed until 1990. A special task force was created in November 1986 within the EARN Board of Directors, with an urgent mandate to propose funding scenarios for 1988 and beyond.

In any case, the change in EARN's funding, and the general increase of X.25 traffic, means that IIEP must plan for substantial increases in data communications budgets in the coming two years. 


\section{CONCLUSIONS}

Networking progress in the last two years can be summarised in the following points:

- emergence of Ethernet as the favoured LAN

- emergence of DECnet and TCP/IP as the de facto LAN protocols

- precursors of truly distributed computing systems (RPC) and file systems

- dramatic growth in WAN infrastructure and traffic

- unfortunate degree of protocol diversity on WANs, with DECnet and RSCS in the lead

- early usage of OSI protocols on LANs and WANs; political committment to OSI

- usage of gateways to palliate diversity

- heavy dependency of LEP collaborations on networking

Such progress has not been without problems:

- protocol diversity

- difficulty of LAN-WAN interconnection

- unusual difficulty of network management and support (protocol diversity multiplied by organisational diversity)

- shortage of skilled people

- high tariffs supported by legal monopolies

- poor quality of service on some international X.25 routes

What lessons can be drawn in support of future planning?

- Since the greatest enemy is diversity, HEP should minimise the number of protocols in use. This could be achieved by minimising the number of computer manufacturers, or by selecting international standard protocols. The first option is politically and economically inappropriate; thus the second should be chosen. (This is not a new policy, but it is now backed up by industrial products and by the general trends in government and academia.)

- A corollary is that HEP should make efforts to hide or eliminate proprietary or national protocols (whenever there is a viable international alternative).

- The success of both home-made and industrial gateways has shown the power of this technique as a palliative for diversity.

- Network support and management is a sink for skilled manpower, and HEP (especially in the smaller European countries) must combine its efforts with the rest of the academic and research community.

Finally, and speculatively, what trends may be predicted for the coming years?

- Useable implementations of a subset of the OSI standards will appear, driven by industry and government. It will become realistic to write such implementations into procurement requirements.

- Political process will favour OSI rather than national or proprietary protocols, and a European management structure for research networking will slowly appear.

- The DECnet and TCP/IP worlds will start to migrate to OSI; RSCS will diminish; SNA will continue, but IBM will also support OSI. 
- Meanwhile the physicists will continue to use many different solutions, so gateways will remain of prime importance for at least five years.

- OSI will "fix" the problems of current (third-generation) networking. However, techniques for true distributed computing, networking of personal computers, and PTT digital services including ISDN, will re-create the problem of diversity.

\section{Acknowledgements}

The listed authors of this paper are limited to direct contributors to its text or content. However, we have reported on the collective work of the CERN Communications Systems group, of many other CERN staff, and of innumerable colleagues in research institutes and universities throughout Europe. It is our duty and pleasure to acknowledge their invaluable contributions. 